\title{
PROJETO PARAMÉTRICO COM FRACTAIS NO DETALHAMENTO DE UMA FACHADA
}

\section{FRACTAL PARAMETRIC DESIGN OF A FAÇADE DETAILING}

\author{
Maycon Sedrez \\ Architect, MSc, PhD Candidate, School of Civil Engineering, Architecture and Urban Design, University of Campinas \\ maycon@fec.unicamp.br
}

Rafael de Moraes Meneghel

Information Technologist, rafael@acertprojetos.com.br

\begin{abstract}
Parametric design and digital fabrication are becoming an ubiquitous tool to contemporary architecture and implies a different method of detailing. With this new perception of the contemporary detail we have developed a façade sun shade using fractal geometry as a generative system. Fractals are complex shapes generated with simple rules, so is relatively easy to change the final geometry when we work with parameters. The design process made possible the creation of many solutions using parametric definitions. Those solutions were physically modeled using rapid prototyping which we consider as an essential tool to the design process. This method also involved analysis and testing of the design using software and the physical models. With this project it was possible to conclude that when working with digital fabrication architects need to incorporate a new set of skills, and that collaboration between professional is extremely important.
\end{abstract}

Keywords: fractal, design process, generative system, digital fabrication.

\section{Introdução}

Desde os anos 70, o projeto de arquitetura vem sofrendo mudanças exigidas pela complexidade dos programas arquitetônicos e a necessidade de aceleração no processo de projeto. Recentemente a incorporação de algoritmos no processo de projeto de arquitetura vem se tornando cada vez mais comum (TERZIDIS, 2006, KOLAREVIC, MALKAWI, 2005). Os algoritmos podem ser gerados utilizando a programação computacional textual ou visual e permitem ao projetista estabelecer um procedimento rigoroso que pode ser aplicado desde a concepção até o detalhe. Isto possibilita ao arquiteto conhecer melhor o problema e descobrir novos métodos para resolvê-lo. O projeto paramétrico viabiliza uma rápida exploração de soluções, pois é relativamente fácil alterar os dados de entrada no código e demais parâmetros. Além disso, o arquiteto adquire um controle maior sobre os modelos projetados analisando-os desde a etapa inicial (KOLAREVIC, 2003).

O detalhe na arquitetura contemporânea sofreu alterações no modo como é compreendido e desenvolvido nas últimas décadas (MOUSSAVI, KUBO, 2006; PICON, 2013). Durante o período Modernista o detalhe frequentemente era projetado de maneira a se ocultar (FORD, 2003). O detalhe contemporâneo assume um papel central no desenvolvimento do projeto e se torna ainda mais expressivo quando auxiliado pela fabricação digital. Picon (2013) complementa que "[t]he proliferation of developments in design software has enabled architects to experiment afresh with texture, colour, pattern and topology". Emmitt, Olie e Schmid (2004:16) também afirmam "[p]resently we are experiencing an enormous amount of technological development, a race towards complete automation [...]", especialmente com a utilização de recursos CAD e CAM na especificação de detalhes arquitetônicos. A fabricação digital permite a produção de peças diferentes com eficiência semelhante à produção de peças padronizadas. Os materiais e os equipamentos de fabricação estão mais presentes também no processo de projeto demandando dos arquitetos um detalhamento específico. Outra característica do detalhe contemporâneo é a criação de edifícios que respondem eficientemente a condições climáticas (PICON, 2013).

Neste contexto a disciplina Prototipagem e Fabricação Digital do programa de pós-graduação em Arquitetura, Tecnologia e Cidade da Unicamp, lecionada pela professora Gabriela Celani, propôs como problema de projeto encontrar uma solução para a fachada curva do edifício CB3. Este edifício será construído na Universidade Estadual de Campinas e incluirá laboratórios multidisciplinares, salas multiuso e espaços de convivência. O objetivo do trabalho foi explorar a geometria fractal de modo paramétrico para estudar o problema de arquitetura acima descrito. Neste artigo os autores explicam o processo de elaboração de um modelo que responde às condições do edifício endereçando o detalhamento. Trata-se de uma pesquisa exploratória que procura abordar a utilização de ferramentas computacionais e fabricação digital para a análise, síntese e avaliação do projeto. 


\section{Materiais e Métodos}

\section{Geometria Fractal}

A geometria fractal surge da investigação cientifica realizada pelo matemático Mandelbrot no final da década de 70. Mandelbrot (1983) procurava uma aproximação da geometria com formas da natureza. Por meio de um sistema de progressão de similaridade subdivido infinitamente pode-se gerar formas fractais. Uma das características relevantes dessa geometria é a de que regras simples geram objetos muito complexos. A utilização de geometria fractal em arquitetura começa desde os anos 80 (JENCKS, 2002), contudo padrões fractais intuitivos foram identificados em períodos anteriores (OSTWALD, 2009). Uma das contribuições dos fractais para a arquitetura é a criação padrões geométricos com regras generativas.

Outros arquitetos já usaram procedimento semelhante em projetos considerados referenciais para esta pesquisa: SOM (KATZ, 2012), Daniel Libeskind, Heneghan Pen, Jean Nouvel e Lab Architecture Studio (BURRY, BURRY, 2010). Em geral os fractais podem se desenvolver em infinitas iterações, mas limitar esse número foi a maneira indicada para aplicar essa geometria no projeto como nos casos acima citados.

\section{Recursos Computacionais}

Dentre os programas utilizados o Rhinoceros ${ }^{2}$ serviu para a modelagem geométrica do edifício, usando como recurso paramétrico o aplicativo para programação visual Grasshopper $^{3}$. O edifício ainda não foi construído, portanto havendo a necessidade de ajustar os parâmetros do projeto, isto pode ser feito facilmente. Essa mesma situação ocorreu com o processo de projeto paramétrico do Aviva Stadium (SHEPERD, HUDSON, HINES, 2011), no qual a execução da obra demandou alterações no projeto.

O Grasshopper possui aplicativos que foram importantes para esta pesquisa: o Geco ${ }^{4}$ e o Hoopsnake ${ }^{5}$. O Geco comunica os dados do modelo com o Autodesk Ecotect ${ }^{6}$. Este add-on funciona como uma interface capaz de enviar dados do modelo para o Ecotect que possibilita análises de iluminação de insolação do edifício. As análises retornam ao Grasshopper e esses dados podem ser utilizados para a definição da geometria. O Hoopsnake repete um procedimento algorítmico a partir de um número definido de iterações. $\mathrm{O}$ componente cria uma cópia do dado recebido do usuário e armazena esse dado localmente. Esse dado duplicado é disponibilizado por meio de um parâmetro de saída padrão.

\section{Forma do Edifício}

As formas do edifício foram elaboradas pelo escritório Modelo da Unicamp atendendo ao plano de expansão da universidade e seguem a geometria curva presente nos edifícios do ciclo básico. Notou-se que o controle da insolação deveria ser estudado com atenção e que qualquer solução para a proteção solar deveria permitir a ventilação de maneira eficiente. A fachada curva do edifício voltada para posição sudoeste resultou em áreas de menor incidência solar e outras com maior necessidade de proteção conforme a estação do ano. Essa primeira constatação evidenciou que uma proteção solar uniforme na fachada inteira não atenderia com eficiência à variação da insolação.

\section{Desenvolvimento}

O processo de projeto seguiu uma sequência comum ao modelo de desempenho sugerido por Oxman (2008), onde a etapa de desempenho e simulação ocorre antes da geração da forma (Figura 1). No modelo convencional o desempenho é testado sobre um modelo gerado previamente. No modelo de desempenho a primeira etapa é a utilização de programas para verificar o desempenho do projeto que informam a definição da forma. A parametrização, nesse caso, contribui para o fluxo do projeto.
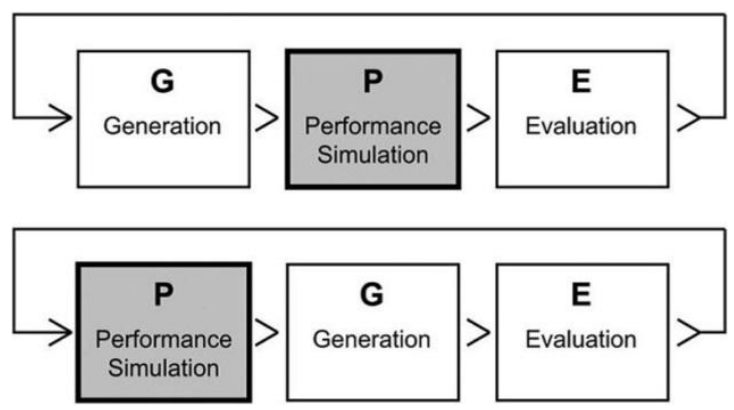

Figura 1. Modelo de desempenho: simulação em CAD convencional (superior) e modelo de desempenho (inferior) (Oxman, 2008).

$\mathrm{O}$ partido arquitetônico baseou-se em estudos de caso e referências de proteção de fachadas como o muxarabiê ${ }^{1}$. Buscou-se criar uma trama que atendesse aos critérios ambientais previamente estudados. O muxarabiê é frequentemente construído com uma trama regular, mas decidiu-se utilizar um padrão fractal recursivo que permitisse variações irregulares no desenho. Considerou-se que os elementos da fachada seriam produzidos com técnicas de fabricação digital. O primeiro passo foi estabelecer um modelo paramétrico do edifício para a elaboração das análises.

Tomando o modelo virtual simplificado como base, foram feitas análises no Ecotect. As Figuras 2 a 4 mostram a região sombreada e a área a ser protegida no período crítico das 17 horas no verão e inverno respectivamente. No inverno pode-se observar a diagonal de sombra gerada pelo próprio edifício.
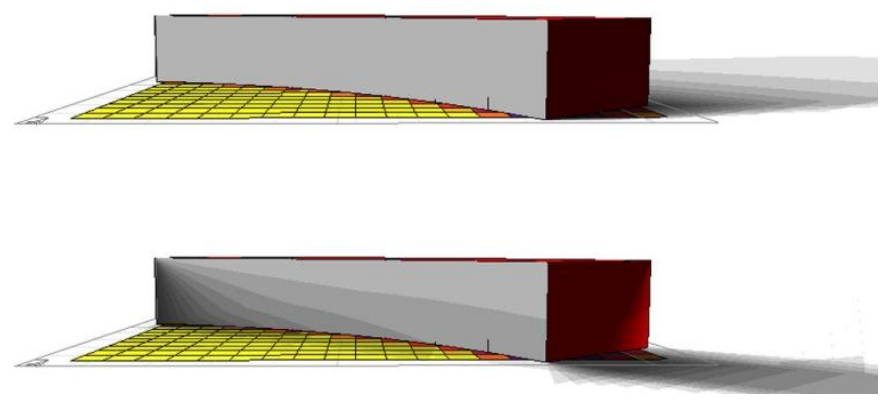

Figura 2. 23 de Dezembro (superior); 23 de Junho (inferior) (Elaboração própria, 2013). 


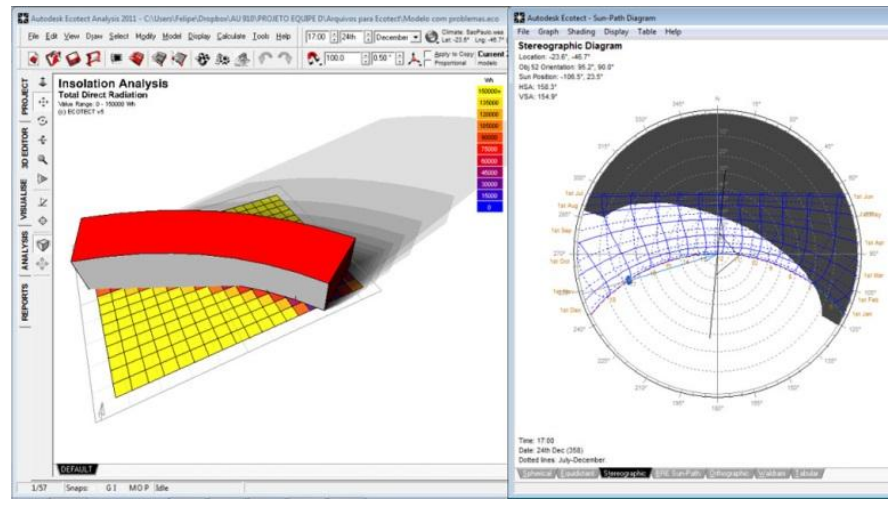

Figura 3. Verão 17 horas (Elaboração própria, 2013).

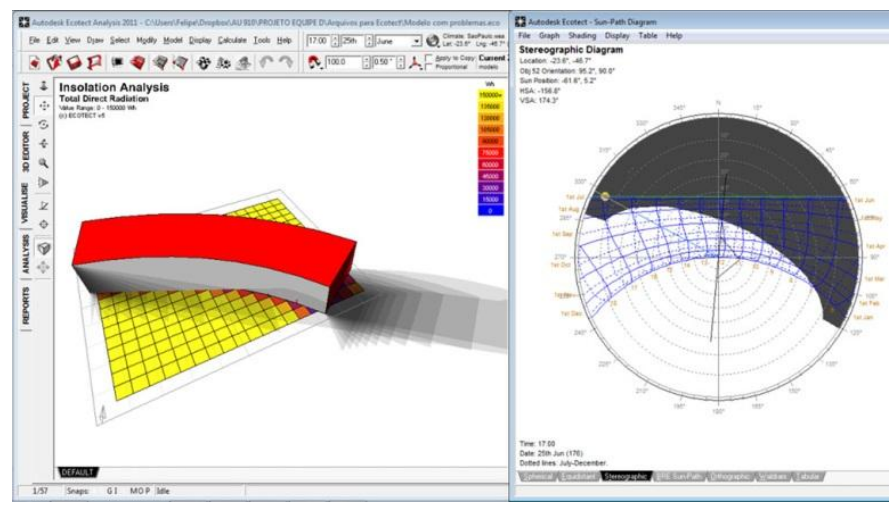

Figura 4. Inverno 17 horas (Elaboração própria, 2013).

O modelo virtual e os arquivos gerais do projeto foram planificados para a produção de desenhos para corte a laser e a elaboração do modelo físico em papel Paraná. Este modelo recebeu as propostas de proteção para a fachada que serão apresentadas adiante. $\mathrm{O}$ modelo físico pôde ser analisado no Heliodon mostrando uma pequena variação em relação às análises numéricas (Figura 5).

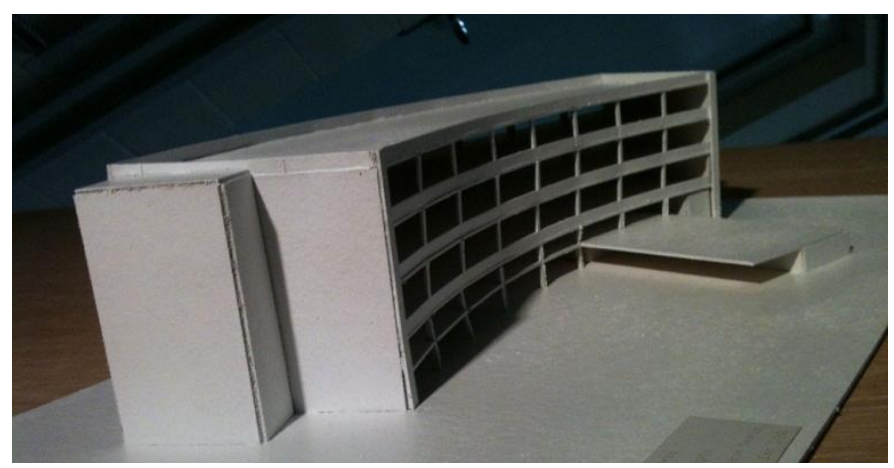

Figura 5. Foto do modelo no Heliodon: Verão 17 horas (Elaboração própria, 2013).

Concluídas as análises de insolação e a construção do modelo, se discutiu a proposta para a fachada. A ideia inicial foi a de divisão da fachada em módulos definidos pelos andares e pilares, e também a criação de uma fragmentação na fachada (Figura 6). Com iterações recursivas seria possível dividir em partes menores as áreas em que houvesse maior incidência solar. Para tanto a geometria fractal se mostrou útil, pois permitiu controlar as iterações de elementos em diferentes escalas, criando um padrão autossimilar. A variação obtida foi associada a áreas com maior ou menor necessidade de luz.

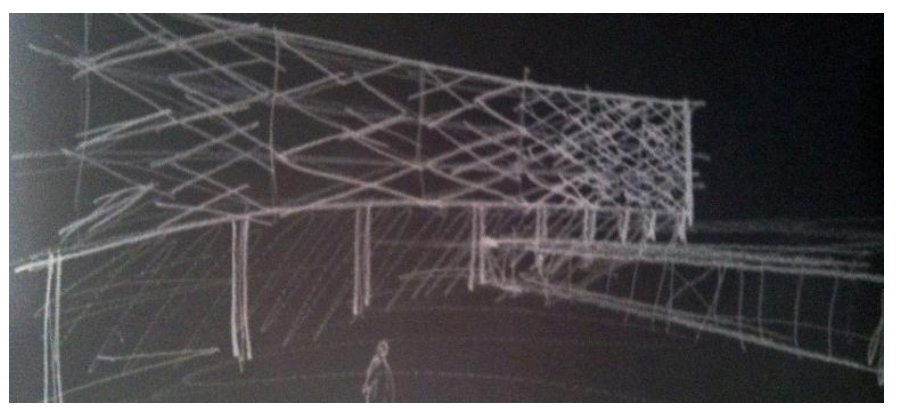

Figura 6. Croqui com a ideia inicial (Elaboração própria, 2013).

Neste momento o projeto ainda estava em um nível abstrato e não se sabia como os elementos do fractal seriam materializados. Porém, qualquer decisão sobre a forma desses elementos poderia ser implementada ou alterada no modelo paramétrico. A primeira proposta para a fachada foi dividi-la em macropainéis a cada andar e a cada eixo estrutural totalizando 24. Como se pode ver na Figura 6 a proposta de divisão criaria diversidade em cada painel em cada andar a cada módulo do edifício e seria controlada pela necessidade de proteção solar. Com isso se atingiria uma variação de desenho para os macropainéis individualmente e haveria uma gama maior de possibilidades para a fixação dos painéis na fachada.

Iniciou-se então a criação de um fractal parametrizado com transformações de rotação e de escala das formas. Os parâmetros originários do modelo virtual informaram pontos em um plano do Rhinoceros. Os pontos serviram como coordenadas para geração do fractal que por sua vez poderia ser associado ao modelo virtual. O processo de projeto paramétrico não é linear e algumas decisões aconteceram simultaneamente, enquanto outras antes ou após as análises. Por exemplo, a decisão de como os painéis para a fachada deveriam ser construídos surgiu em diversos momentos no processo, influenciando as decisões ao longo do desenvolvimento do projeto.

Quatro alternativas de utilização da forma fractal na fabricação dos paineis foram criadas e estão aqui descritas com a nomenclatura em inglês para relacioná-las aos componentes do Grasshopper: lines, pipes, planes e cuts (Figura 7). As lines são as formas iniciais do fractal construídas ligando pontos específicos da fachada e são iteradas a partir de parâmetros definidos por ângulos e eixos de rotação. Os pipes são construídos com o componente do Grasshopper de mesmo nome, e são criados usando as linhas como centro e um raio especificado. A extrusão das linhas em uma direção perpendicular ao plano no qual estão contidas, cria os planes. E, por fim, a subtração das pipes de um plano cria um desenho vazado (Figura 7).

As lines submetidas a transformações criam padrões que foram testados e alterados. Buscou-se uma forma que seguisse a diagonal de sombra no inverno conforme o estudo de insolação. Essa região poderia ser mais aberta em relação às demais. As alternativas planes e pipes foram testadas na forma de um único painel para verificar a projeção de sombra ou luz que o padrão geraria (Figura 8). Nesses testes de 
sombreamento os cuts seriam o negativo dos pipes. Os planes poderiam funcionar como bandeja de luz, porém criaram uma dificuldade de execução. O número de peças de encaixe necessárias seria muito alto, o que aumentaria a dificuldade de montagem e o peso dos macropainéis. Outra condicionante é que nem todos os pontos da fachada poderiam receber algum tipo de fixação.
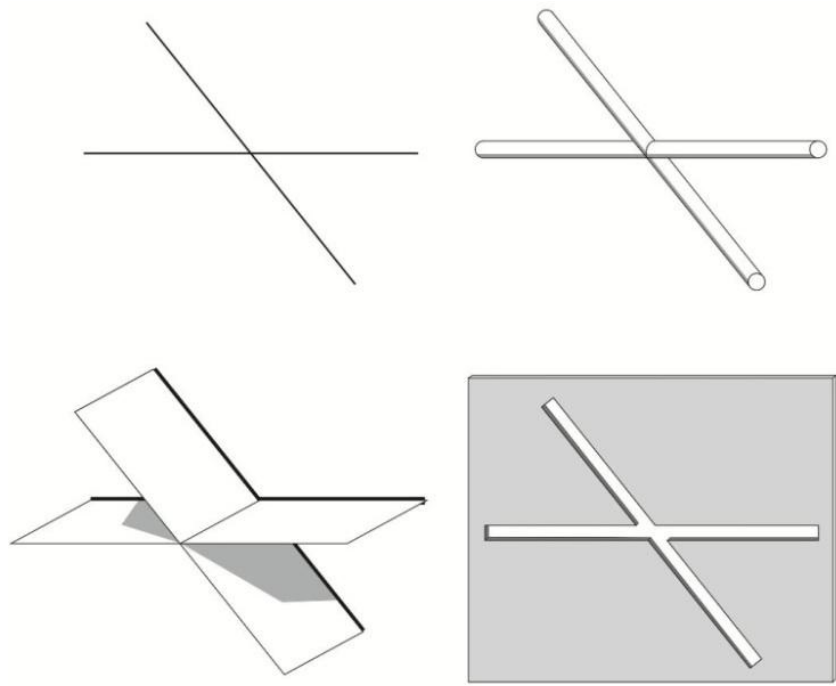

Figura 7. Lines, Pipes (superior); Planes e Cuts (inferior) (Elaboração própria, 2013).

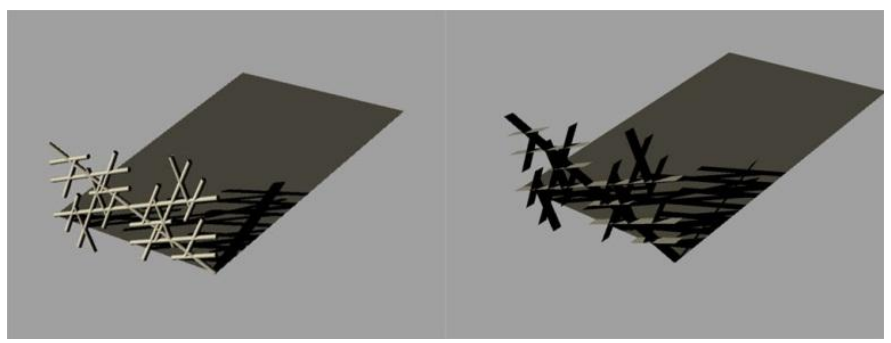

Figura 8. Estudo de sombreamento com pipes e planes (Elaboração própria, 2013).
Analisando as questões construtivas, os planes foram excluídos por envolver muitas peças, soldagem ou encaixes, além disso, a limpeza e manutenção seriam difíceis. Os pipes também foram descartados visto que necessitariam acesso a um maquinário não disponível para curvatura de tubos (calandragem) e de soldagem. $\mathrm{O}$ resultado com pipes seria similar ao projeto Wuzhen Theater de Artech Architects utilizado como referência nos estudos (Figura 9), neste exemplo o elemento não cumpre a função de proteção solar.

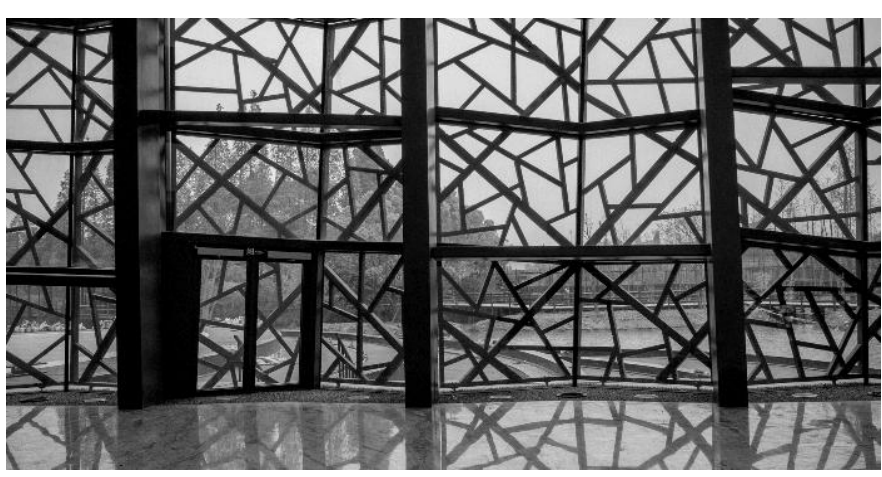

Figura 9. Wuzhen Theater, vista interna (YING, 2010).

Decidiu-se então trabalhar com os cortes dos painéis pela viabilidade de equipamentos e disponibilidade de tempo para produção do projeto e dos protótipos. A primeira proposta foi realizada com transformações específicas e controladas no Grasshopper. No Hoopsnake foi determinado o número de iterações necessárias. Uma definição de fractal foi criada e aplicada, com pequenas variações, em cada um dos 24 painéis (Figura 10).

$\mathrm{Na}$ segunda proposta um fractal único para toda a fachada se espalharia em todos os painéis. Nesse caso foi necessário planificar a fachada. A transformação fractal elaborada estava apoiada inicialmente em uma superfície única, pois este processo não funcionaria em uma superfície facetada ou curva. Posteriormente, esse fractal contínuo foi separado nos 24 painéis, porém mantando a ideia de continuidade do desenho (Figura 11).

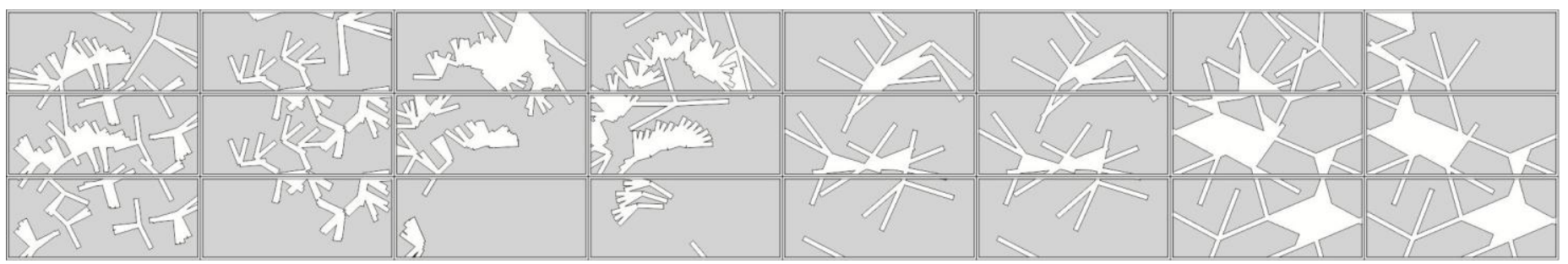

Figura 10. Detalhe dos 24 painéis com um fractal variando para cada painel. (Elaboração própria, 2013).

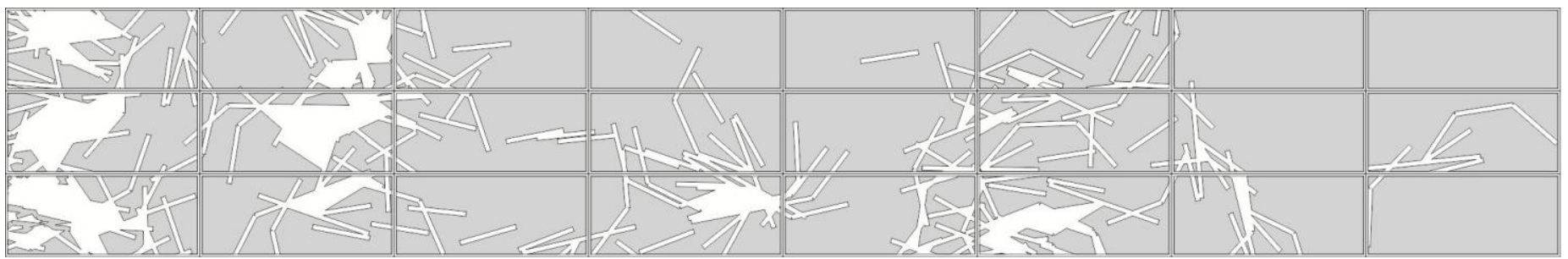

Figura 11. Detalhe dos 24 painéis com um fractal único variando para toda a fachada (Elaboração própria, 2013). 
Em seguida foi analisada a questão de execução dos paineis. Uma primeira questão que poderia dificultar a execução eram as peças que se soltam ou se perdem devido ao cruzamento das figuras (áreas brancas maiores), além da falta de rigidez da chapa provocada pelo essa geometria. Isso foi percebido durante o processo, mas ficou mais evidente quando se produziu um modelo físico em escala, cortado a laser em chapa de papelão. Na Figura 12 observa-se que a peça vermelha seria perdida e que os cantos laranja se tornariam frágeis. Isto foi resolvido com a execução de um detalhe que elimina encontros por meio da criação de uma borda. Este processo poderia ser automatizado, mas foi executado com base em decisões estéticas por isso, nesta etapa o projeto passou por uma fase de desenho manual.
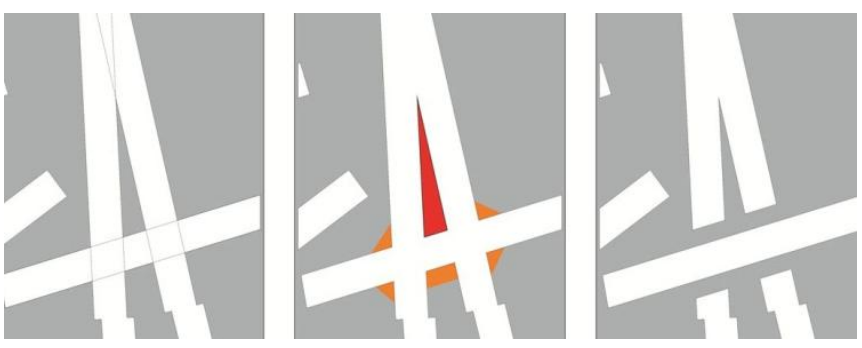

Figura 12. Detalhe do desenho gerado e sua edição (Elaboração própria, 2013).

A passagem de ar ainda estava limitada nas áreas mais fechadas. Então, a partir de outra referência de projeto, The Orange Cube de Jakob e Macfarlane (Figura 13), decidiu-se usar chapas perfuradas. Contudo, cortar as chapas previamente perfuradas não seria a melhor opção. Testes com a máquina CNC (Computer Numerical Control) mostraram que haveria problemas decorrentes da deformação da chapa por causa dos furos. Além disso, as figuras a serem cortadas ficariam com um desenho de bordas irregulares como apresentado na Figura 14.

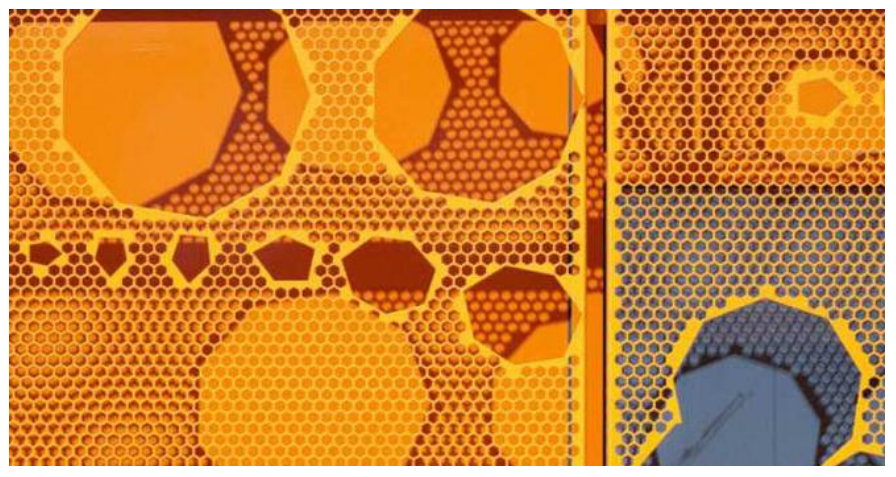

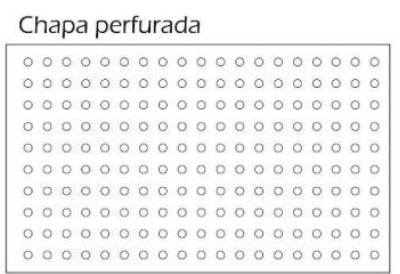

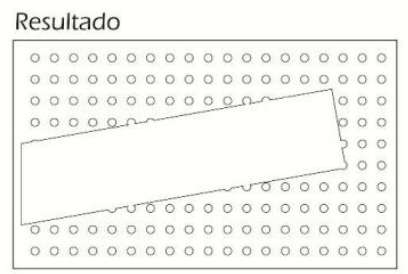

Caminho do corte

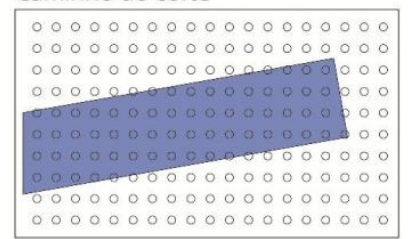

Recorte

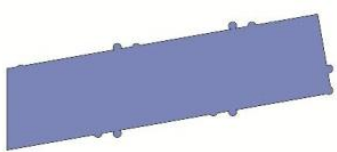

Figura 14. Detalhe do corte de uma chapa perfurada (Elaboração própria, 2013).

A solução encontrada foi cortar as chapas para em seguida perfurá-las. Foi criado um algoritmo de perfuração que exclui os furos de áreas dentro dos cuts e em sua borda. Isso mantém a integridade do desenho planejado e cria resistência na borda dos vazados. As chapas podem ser produzidas por qualquer fornecedor com ferramentas CNC. Na Figura 15 apresenta-se o código de controle da perfuração aplicado em uma parte do projeto; um parâmetro de offset (em laranja) controla a margem que haverá na borda excluindo os furos nesta região, sliders do Grasshopper controlam a quantidade de furos e a sua dimensão.

Havendo solucionado os problemas das partes "soltas", falta de rigidez e da perfuração, foram feitas algumas simulações de variações para a fachada. Por fim optou-se por uma forma inicial que são 11 linhas iniciais construídas sobre uma diagonal que cruza a fachada do canto superior esquerdo até o canto inferior direito. Esta diagonal coincide com a linha da sombra produzida pelo próprio edifício no inverno. No canto inferior esquerdo da fachada há menor incidência solar, portanto essa área pode concentrar mais painéis vazados. Foi selecionada a opção que aparece em vermelho na figura 16.

\section{Resultados}

Com o desenho final definido, ocorreu a etapa de trabalho de ajuste dos cortes. Os modelos foram produzidos em diferentes escalas para se estudar as relações do projeto; com papel em uma máquina de corte a laser e em alumínio em uma máquina CNC.

Figura 13. Detalhe da proteção solar do edifício Orange Cube (BOREL, 2011).
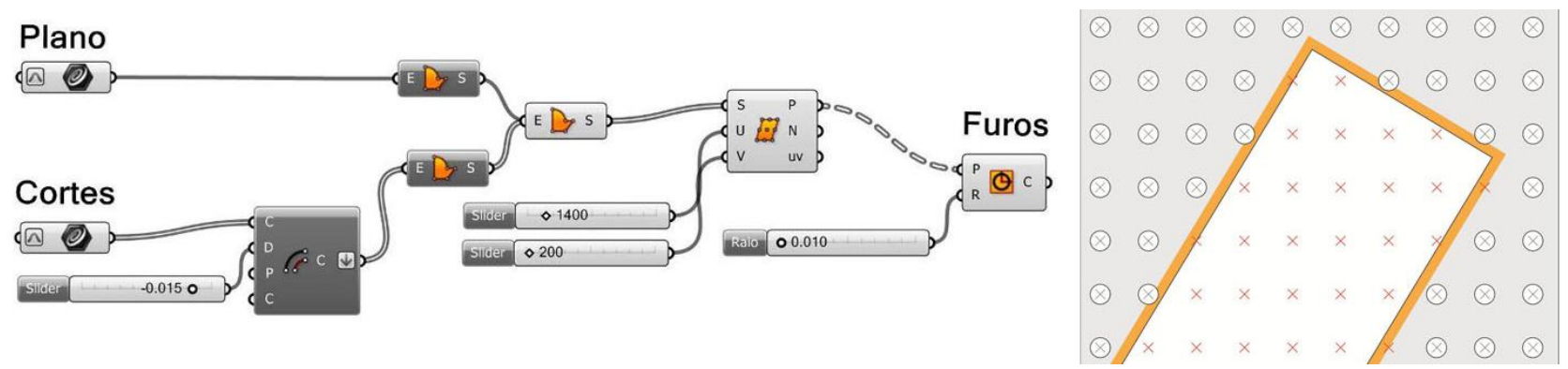

Figura 15. Algoritmo de perfuração no Grasshopper e detalhe da chapa com furos. (Elaboração própria, 2013). 

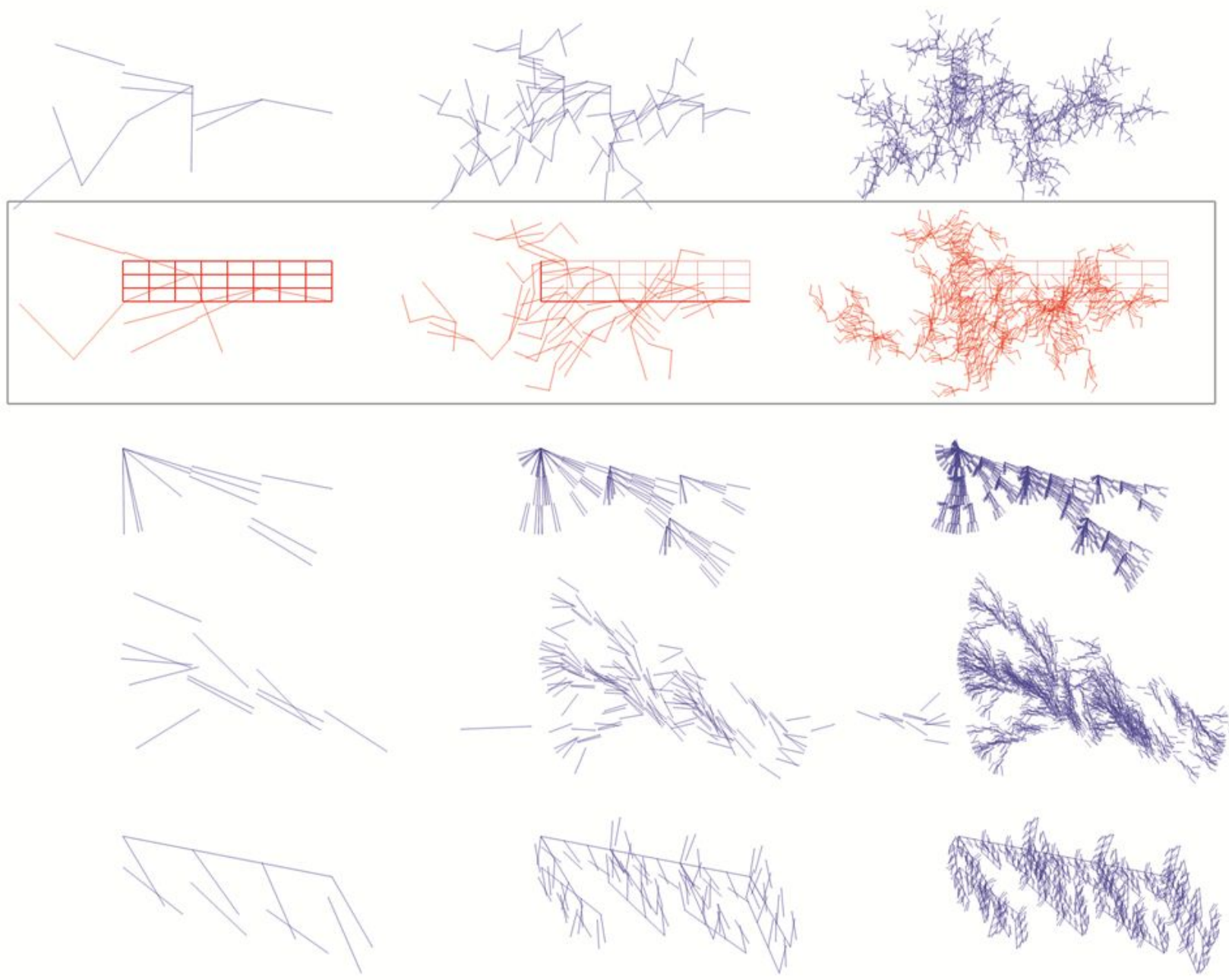

Figura 16. 5 variações de iteração de fractais com diferentes transformações. (Elaboração própria, 2013).

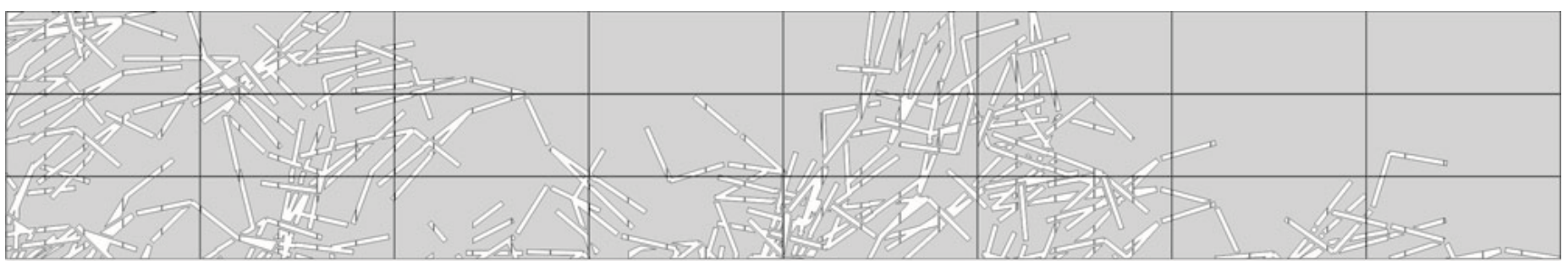

Figura 17. Detalhe da proposta final. (Elaboração própria, 2013).

A proposta final foi desenhada, modelada e testada novamente (Figuras 17 e 18). Cada um dos 24 macropainéis seria dividido em 5 painéis menores posicionados verticalmente totalizando 120 peças. Um detalhe de uma das peças pode ser visto na Figura 19.

A parte final da pesquisa consistiu na elaboração dos detalhes de fixação conforme a especificação de fornecedores e pesquisa de referências de detalhes arquitetônicos para fixação (Figura 20). Os pilares e vigas do edifício receberiam placas de fixação que apoiam montantes parafusados para o encaixe dos painéis. Os detalhes de fixação seguiram padrões geralmente utilizados, mas sofreram alterações necessárias para acomodarem os painéis e se ajustarem à curvatura do edifício.

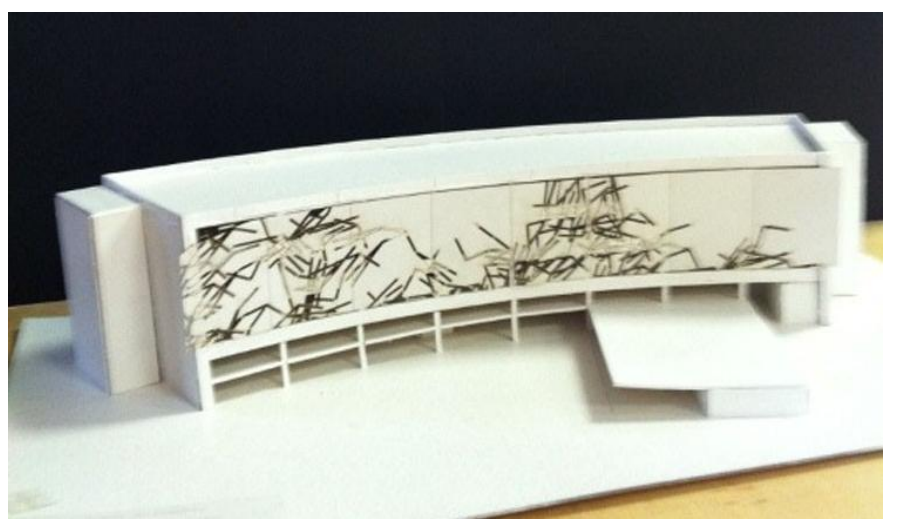

Figura 18. Modelo físico com a proposta final (Elaboração própria, 2013). 


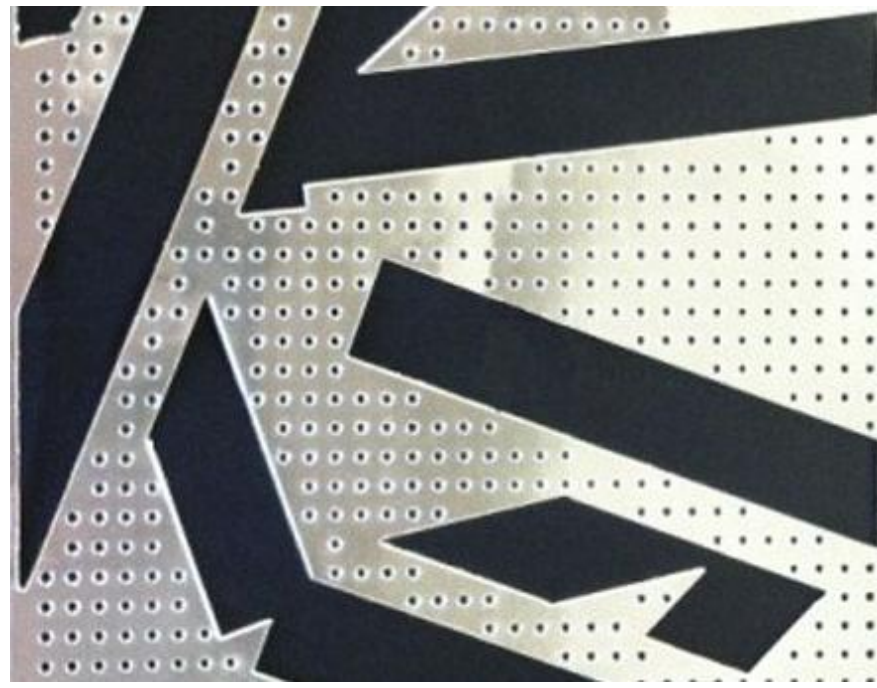

Figura 19. Detalhe de um dos 120 painéis necessários para a fachada alumínio fresado (Elaboração própria, 2013).
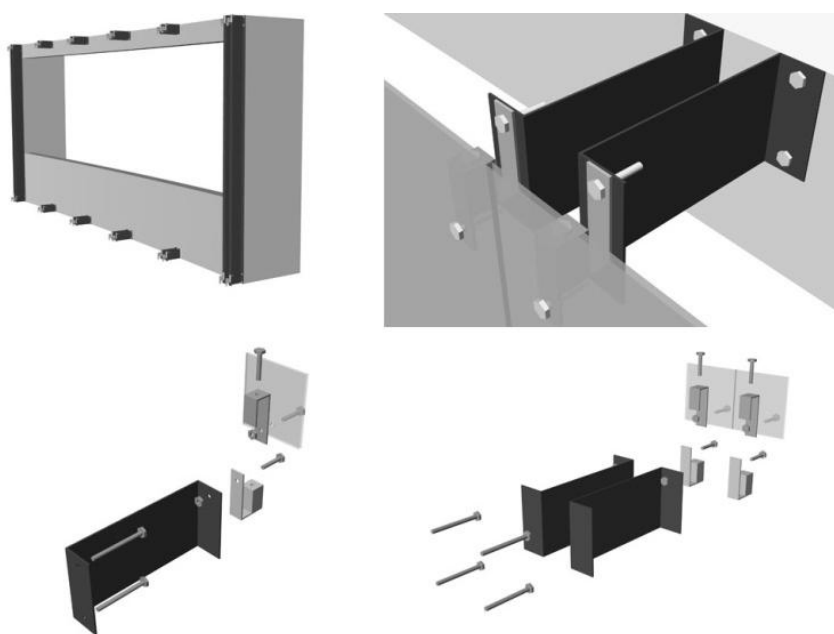

Figura 20. Detalhes de fixação (Elaboração própria, 2013).

Os trabalhos foram finalizados com a realização de renderings (Figuras 21 e 22) de uma das salas do edifício em diferentes períodos do ano, resultando no efeito visual desejado para o interior do prédio e também garantindo uma redução da incidência solar sem redução da iluminação. Posteriormente, com a definição do tipo de esquadrias, esse modelos podem ser novamente testados e alterados se necessário.

\section{Conclusões}

Esta pesquisa descreve como o detalhe em arquitetura se torna uma atividade presente em todas as etapas do processo de projeto quando se usam processos generativos e paramétricos. $\mathrm{O}$ ambiente computacional integrado com a ferramenta de análise térmica torna ágil a análise do edifício e ao mesmo tempo fornece parâmetros para o ajuste da proposta. $\mathrm{O}$ ambiente paramétrico permite a geração de diversas alternativas a partir do mesmo algoritmo por meio de pequenas alterações nos dados. Desta maneira é possível realizar um processo de projeto com a abordagem contemporânea descrita por Tidafi e Iordanova (2006) que sugerem além de explorar diferentes soluções formais, comunicar todo o processo do projeto.

Essas soluções podem ser testadas virtualmente sob diversos aspectos com as tecnologias disponíveis ou testadas fisicamente havendo a disponibilidade de recursos de prototipagem rápida. É importante acrescentar que diversos ajustes necessários no projeto só foram percebidos com a produção dos modelos, como alterações de medidas gerais, espaçamento adequado de perfuração e criação de bordas de segurança de corte. A continuação deste processo levaria à criação dos arquivos prontos para produção (file-to-factory) com ferramentas $\mathrm{CNC}$ e a produção de mais alguns protótipos eliminaria qualquer problema de ajuste. Embora esta parte do trabalho tenha sido desenvolvida colaborativamente entre um especialista em arquitetura e outro em computação, após visitas a empresas de fabricação de paineis de fachada foi possível notar que a finalização do processo exigiria a colaboração entre mais profissionais ainda, envolvendo provavelmente engenheiros e fabricantes de paineis metálicos.

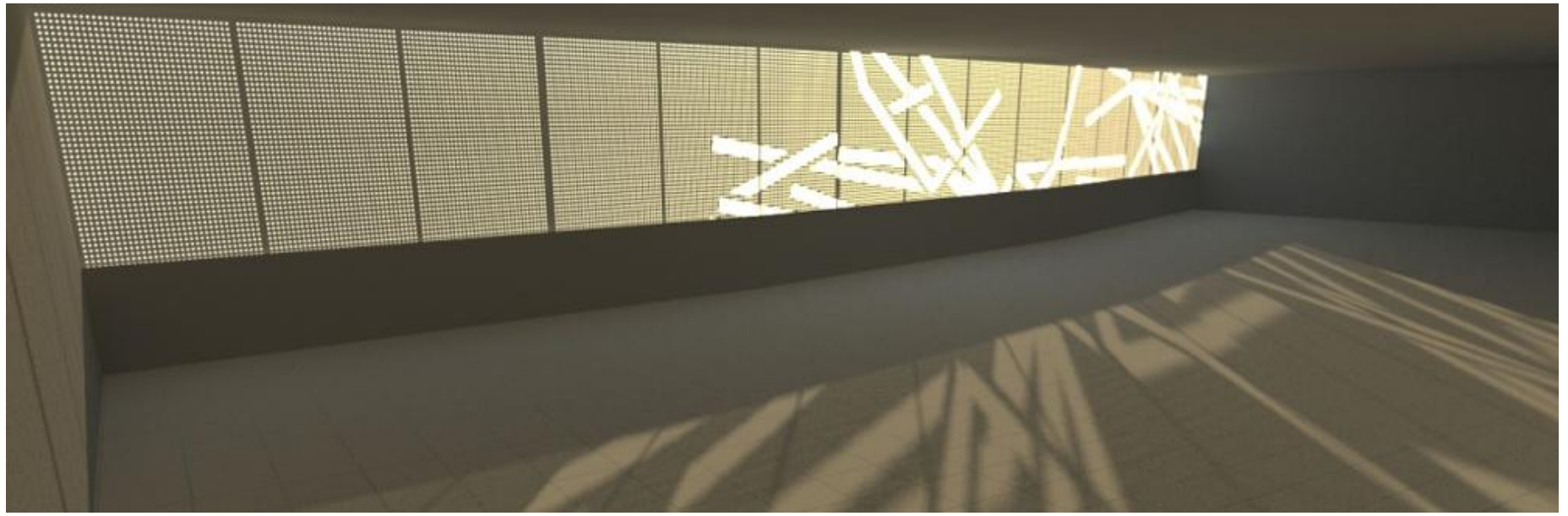

Figura 21. Detalhe da projeção da luz no interior no verão. (Elaboração própria, 2013). 


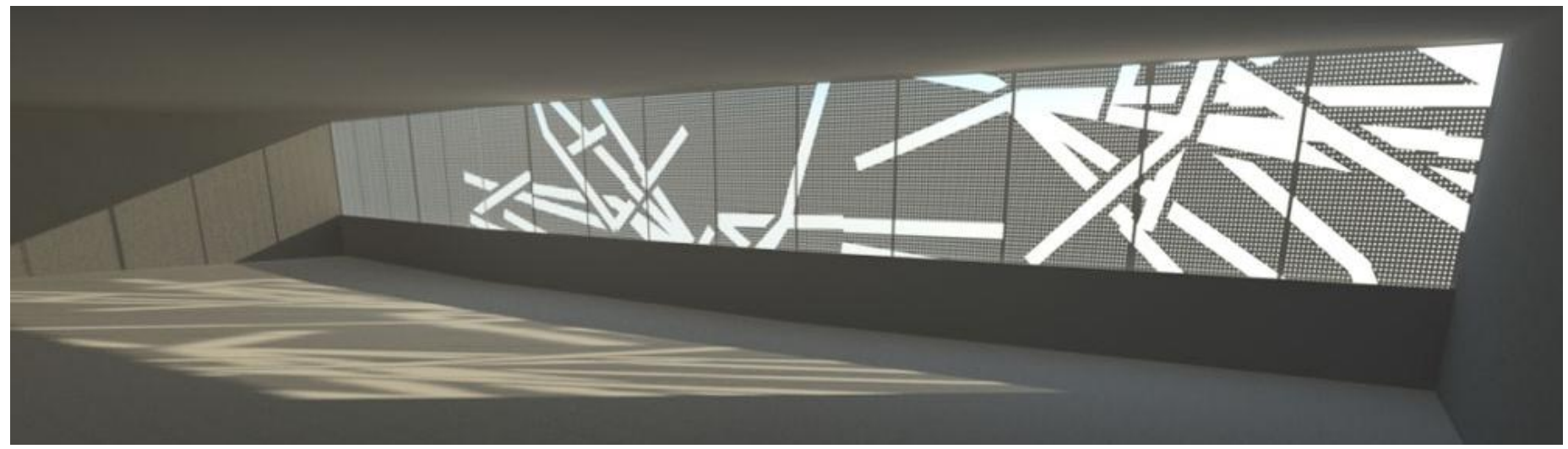

Figura 22. Detalhe da projeção da luz no interior no inverno. (Elaboração própria, 2013).

\section{Agradecimentos}

Agradecemos à CAPES pela bolsa de doutorado do pesquisador Maycon Sedrez. Agradecimentos também aos alunos de graduação Maja Zeller, Felipe Tricoli Jardim e Yumi Neder, que participaram deste projeto.

\section{Notas}

(1) O muxarabiê ou muxarabi é um tipo de proteção para janelas originária da arquitetura árabe, geralmente construída com uma treliça de madeira.

(2) Rhinoceros é um programa de CAD (Computer-Aided Design).

(3) Grasshopper é um plug-in para Rhinoceros de modelagem geométrica por meio de programação.

(4) Geco é um conjunto de componentes que estabelecem uma conexão direta entre Rhinoceros e Grasshopper com o Ecotect.

(5) Hoopsnake é um add-on para Grasshopper que permite a criação de algoritmos iterativos.

(6) Ecotect é um programa da Autodesk para análise de insolação, sombreamento e desempenho térmico.

\section{Referências}

BOREL, N. The orange cube. 2011. 1 fotografia, color. Available in: < http://www.archdaily.com/111341/the-orange-cube-jakobmacfarlane-architects/> Access in: 19 set. 2013.

BURRY, J.; BURRY, M. The new mathematics of architecture. New York: Thames and Hudson, 2010.

EMMITT, S.; OLIE, J.; SCHMID, P. Principles of Architectural Detailing. Oxford: Wiley-Blackwell, 2004.

FORD, E. The details of modern architecture. Cambridge: The MIT Press, 2003.

JENCKS, C. The new paradigm in architecture: the language of post-modernism. New Haven: Yale Press University, 2002.

KATZ, N. Façade pattern through scripting: recursion. In: Leach, Neil; Yuan, Philip F. Scripting the future. Shanghai: Tongji University Press, 2012.

KOLAREVIC, B; MALKAWI, A. M. Performative architecture: beyond instrumentality. New York: Spon Press, 2005.

KOLAREVIC, B. Architecture in the digital age: design and manufacturing. New York: Spon Press, 2003.

MANDELBROT, B. The fractal geometry of nature. New York: W.H. Freeman, 1983.

MOUSSAVI, F.; KUBO, M. The function of ornament. Barcelona: Actar, 2006.

OSTWALD, M. Fractal Architecture: knowledge formation within and between architecture and the sciences of complexity. Saarbrücken: VDM Verlarg, 2009.

OXMAN, R. Digital architecture as a challenge for design pedagogy: theory, knowledge, models and medium. Design Studies, no. 29, 2008, p. 99-120.

PICON, A. Ornament: the politics of architecture and subjectivity. AD Primer. London: Wiley, 2013.

TERZIDIS, K. Algorithmic Architecture. London: Elsevier, 2006.

TIDAFI, T..; IORDANOVA, I. Experimental Approach in an Architectural Design Studio - How Digital Technologies Could Change a Design Process. Communicating Space(s) - 24th eCAADe Conference Proceedings. Volos (Greece) 6-9 September 2006, pp. 852-858. Anais... 2006. Available in: <http://cumincad.scix.net/cgi-bin/works/Show?_id=2006_852>. Access in: 28 jan. 2013.

SHEPERD, P.; HUDSON, Roly; HINES, David. Aviva Stadium: a parametric success. International Journal of Architectural Computing, ed. 2, v. 9, 2011.p. 167-186.

YING, F. Wuhzen Theater. 2010. 1 fotografia, p\&b. Available in: < http://www.archdaily.com/375609/wuzhen-theater-kris-yaoarchitect/> Access in: 19 set. 2013. 